\title{
Some problems with reaction time as a measure of memory search
}

\author{
ROBERT LEWIS DURHAM and DORIS SESTOKAS \\ University of Colorado, Colorado Springs, Colorado 80907
}

\begin{abstract}
Previous studies of memory search, utilizing reaction time (RT) as an index of processing time, have indicated faster RTs for a probe stimulus which is present in memory set than for one which is absent. Dodson (1972) reported a crossover interaction involving RTs when Ss responded with dominant or nondominant hand to stimulus presence or absence, thus confounding the above mentioned main effect. The present study replicated the interaction reported by Dodson utilizing a different RT measure on a task not involved with immediate memory search. One of three colors (red, green, or blue) was randomly designated the positive color in the experiment for each S. Since all three colors are assumed equally available in memory, differences in RTs may not be accounted for in terms of encoding strategies. Rather, they must be due to differences in response selection. Results indicated that left-handed females' RTs accounted for the variance associated with this interaction. Results were discussed in terms of a response selection bias for left-handed Ss.
\end{abstract}

In recent years, studies concerning information processing and memory scanning have been investigated extensively (e.g., Sternberg, 1966; 1967; 1969). A dependent measure typical of these studies has been reaction time (RT), where Ss respond to the presence or absence of a probe stimulus in a memory set of items (e.g., Theios, Smith, Haviland, \& Traupmann, 1971). RT has been utilized to indicate the type of search procedures (e.g., self-terminating, exhaustive, etc.) which Ss employ as well as the absolute rate of per item search.

Not only are absolute RTs important as indicators of the speed of memory search, but also interactions of independent variables (e.g., size of memory set and position of probe stimulus in memory set) are utilized to infer the type of search process engaged in by Ss. Thus, the interaction between variables influencing RT as well as the absolute amount of time involved in information processing are of both theoretical and practical importance.

Dodson (1972), investigating the effects of repetition of memory set items on memory search, reported findings which were generally congruent with the existing body of literature concerning memory search. However, he also reported a curious crossover interaction response type (presence or absence of probe stimulus) and handedness (whether $S$ was responding with his dominant or nondominant hand to the probe stimulus). This interaction indicated that Ss responded more quickly to a probe stimulus in memory set with their D hand but faster to a probe stimulus not in memory set with their ND hand. This result differed from the usual situation of faster RTs when detecting the presence rather than the absence of a probe stimulus. Dodson did not discuss this interaction or its relationship to the main effect.

In reviewing other literature concerning $\mathrm{RT}$ as a measure or memory search, it was noted that rarely was the absolute handedness (right or left) of Ss included as a factor in the analyses. Usually experiments were counterbalanced, so that $\mathrm{S}$ was allowed to respond to the presence or absence of the probe stimulus with both hands over the couse of the study. Since absolute handedness has been shown to interact with task variables such as hand efficiency in other areas of research (e.g., Rigal, 1974), the present study was initiated to examine the possibility that handedness might account for the interaction reported by Dodson (1972).

The present study had two objectives: (1) to demonstrate the generality of the interaction reported by Dodson, by replicating his results with a different RT measure (key depression as opposed to key release) on a task not involved with immediate memory search and (2) to uncover the source of this interaction.

The task in the experiment was not concerned with encoding new material. Rather, it was concerned with identifying stimuli which were assumed equally available in memory. It was proposed that response selection, rather than encoding strategies, should account for the interaction demonstrated by Dodson. In the present study, a colored light (red, green, or blue) was randomly designated the positive color for each $\mathrm{S}$. The other two stimulus colors were assumed to be indicants of stimulus absences. Since the positive color was randomly assigned, it was assumed that the colors were equally available in memory for the recognition task. Hence, any differences in RTs should be attributable to response selection and not encoding differences between Ss.

A tentative hypothesis was proposed to account for the interaction on the basis of S sampling. It would be expected that most Ss are right-handed. Consequently, the interaction should be attributable to the responses elicited by right-handed Ss. It was, therefore, hypothesized that right-handed Ss have a predisposition 
to respond to any positive ("correct") stimuli with their dominant hand and tend to be slightly quicker with their nondominant hand to a negative (incorrect) stimulus. The source of the interaction should, therefore, be attributable to a response selection bias and not a stimulus identification factor.

\section{METHOD}

\section{Subjects}

Ss were 40 undergraduate volunteers (average age $=24.8$ ) enrolled in psychology courses at the University of Colorado at Colorado Springs. There was no compulsorary experimental participation requirement. Unknown to the $\mathrm{E}$ (the second author), $20 \mathrm{Ss}$ were left-handed and 20 were right-handed. Handedness was defined as that hand with which the $S$ wrote. There were an equal number of males and females in each group. In addition, 5 of the $10 \mathrm{Ss}$ in each subcell were randomly assigned to one of two stimulus presentation orders.

\section{Design and Apparatus}

The experiment was conducted utilizing a Lafayette 63012 multichoice reaction time apparatus. With this device, colored lights (red, green, or blue) are presented as stimuli. The stimuli were presented on the stimulus box, located approximately $.5 \mathrm{~m}$ directly in front of S. S could respond to the stimulus by depressing either of two telegraph keys. The telegraph keys were between $\mathrm{S}$ and the stimulus box.

One of the three colors was randomly assigned to each $\mathrm{S}$ as his positive (correct) color. The remaining two colors were defined as examples of the absence of the positive dimension. Every $10 \mathrm{sec} \mathrm{S}$ was presented a stimulus. Two blocks of trials with 25 stimulus presentations in block were administered. The first five trials of each block were designated as practice trials and were not analyzed. As mentioned previoulsy, Ss were counterbalanced so that one-half of the Ss responded to the positive stimulus with their dominant hand during the first block and one-half with their nondominant hand. In the second block of 25 trials $S$ responded to the positive color in the first block. In each block of 20 trials, 10 stimuli were positive and 10 negative. The stimuli were presented randomly with the restriction that no more than three consecutive trials could elicit the same response. The result was a 2 by 2 by 2 by 2 by 2 by 10 analysis of variance design. Factors $\mathrm{A}$-handedness, $\mathrm{B}$-sex, and $\mathrm{C}$-order of presentation were between-Ss factors and Factors D-dominance, $\mathrm{E}$-stimulus presence, and $\mathrm{F}$-trials were within-Ss factors.

\section{Procedure}

Each $S$ was run individually through the experimental procedure. Each experimental session lasted approximately $15 \mathrm{~min}$. After familiarizing $\mathrm{S}$ with the apparatus, $\mathrm{S}$ was instructed as to his role in the study. $S$ was told that the experiment was involved with reaction time, and that he would see either a red, green, or blue stimulus every $10 \mathrm{sec}$ throughout the course of the study. He was told that _____ (for example, red) was the correct stimulus, and that he was to depress the key with his hand (for example, right) every time he saw that color. Any other color which appeared was to elicit a response from the other hand. For example, a right-handed male was randomly assigned the color blue as the positive color. He was in the cell which had him respond to positive stimulus presence with his nondominant hand in the first block of trials. He was told that blue was the correct stimulus color. Each time he saw the blue light, he was to depress the left key. When any other color (red or green) appeared, he was to indicate his response with his right hand.

It was emphasized that the positive color was correct, and that $\mathrm{S}$ was to respond as quickly as possible, without making errors, when the stimuli appeared. After answering any questions concerning the experimental procedure, the first block of five practice and 20 test trials was initiated. Following the first block, $S$ was given a brief rest. $S$ was then informed that the next half of the study was slightly different from the first, in that now $S$ was to respond to the positive color with his other hand. It was noted that the color was still the same and that the only difference was reversing S's hands in terms of indicating the correct and incorrect stimulus. E then administered the final 25 trials. Following the experiment, E answered any questions and verified the handedness of each $\mathrm{S}$.

\section{RESULTS}

Due to computer program restrictions, the initial ANOVA combined the factors of dominance and stimulus presence into one factor. In this initial analysis, RT was analyzed in a 2 by 2 by 2 by 4 by 10 analysis of variance. Factors $\mathrm{A}, \mathrm{B}$, and $\mathrm{C}$ were the same between-Ss factors (handedness, sex, and order of presentation) described above. Factor D was the combination of the dominance and stimulus presence factors. Factor $\mathrm{E}$ was trials. Both Factors D and E were within-Ss factors. The analysis revealed significant main effects attributable to $\operatorname{sex}[F(1,32)=5.403, p<.05]$ and the combined dominance-stimulus presence factory $[\mathrm{F}(3,96)=5.245$, $\mathrm{p}<.01]$. In addition, two interactions were significant: Sex by Dominance-Stimulus Presence $[F(3,96)=4.115$, $\mathrm{p}<.05]$ and Sex by Handedness by Dominance-Stimulus Presence $[\mathrm{F}(3,96)=4.980$, $p<.01]$.

Since there were no main effects or interactions involving Factor E (trials), this factor was eliminated from subsequent analyses. Median RTs were then subjected to a 2 by 2 by 2 by 2 by 2 ANOVA, without trials as a factor. Factors $\mathrm{A}, \mathrm{B}$, and $\mathrm{C}$ were the same between-Ss factors as noted above. Factor D was dominance and Factor $\mathrm{E}$ was stimulus presence. Both these factors were within-Ss. Results of this analysis indicated main effects attributable to sex $[F(1,32)=$ $5.415, \mathrm{p}<.05]$ and stimulus presence $[\mathrm{F}(1,32)=6.908$, $\mathrm{p}<.05]$. The mean RT for males was $459 \mathrm{msec}$ and for females, $517 \mathrm{msec}$. The mean RT for detecting the positive stimulus was $478 \mathrm{msec}$ and the mean RT for detecting other colors was $498 \mathrm{msec}$.

In addition, there were four significant interactions in this analysis: the Dominance by Stimulus Presence interaction $[F(1,32)=5.373, p<.05]$, the Handedness by Sex by Stimulus Presence interaction $[F(1,32)=$ 4.937, $\mathrm{p}<.05$ ], the Sex by Dominance by Stimulus Presence interaction $[F(1,32)=7.878, p<.01]$, and the Handedness by Sex by Dominance by Stimulus Presence interaction $[F(1,32)=6.212, p<.05]$. These interactions are depicted in Fig. 1, 2, 3, and 4.

To determine the source(s) of the significant interactions, four additional analyses of variance were performed on the data. A separate analysis was performed for sex by handedness between-Ss factors. Thus there was a 2 by 2 by 2 ANOVA performed for 


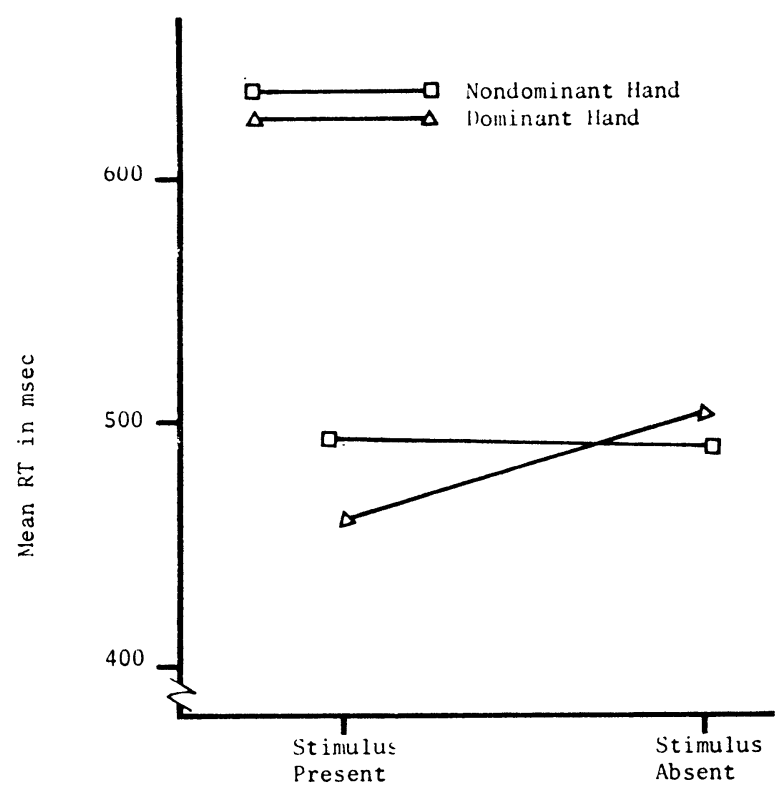

Figure 1. - Mean RT to the presence or absence of probe stimulus in memory set with dominant and nondominant hands.

right-handed males, right-handed females, left-handed males, and left-handed females. Factor A-order of presentation was a between-Ss factor and Factor $\mathrm{B}$-dominance and $\mathrm{C}$-stimulus presence were within-Ss factors in all four analyses. Except for main effects attributable to stimulus presence, the only significant main effect or interaction was in the analysis of left-handed females. This interaction was between stimulus presence and dominance $[\mathrm{F}(1,8)=12.298$,

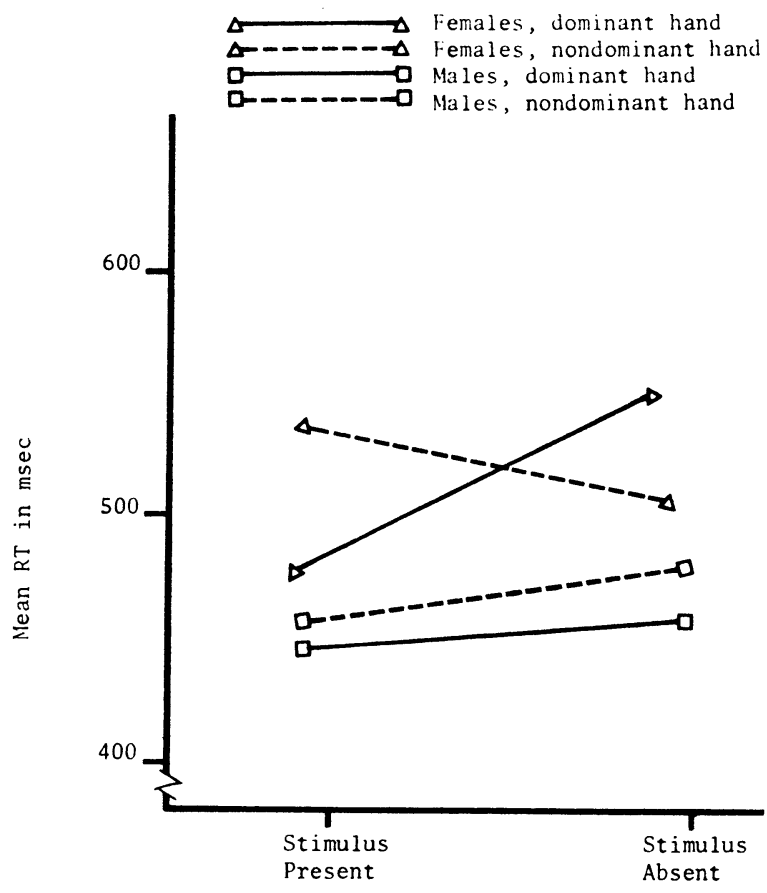

Figure 3. - Mean RT to the presence or absence of probe stimulus in memory set by sex and dominance of hand.'
REACTION TIME AND MEMORY SEARCH

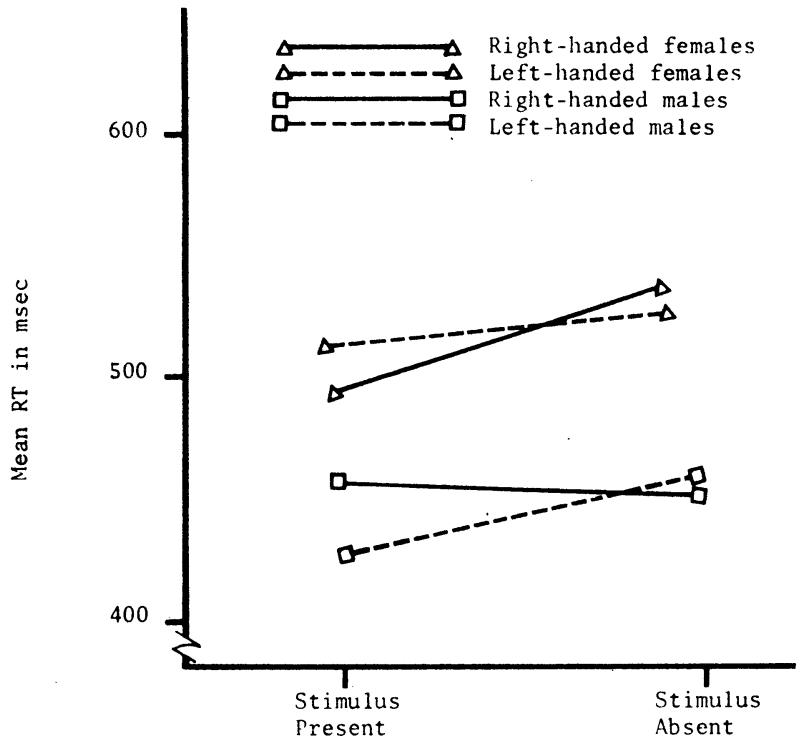

Figure 2. - Mean RT to the presence or absence of probe stimulus in memory set by sex and handedness.

$\mathrm{p}<.01]$. This interaction is indicated as a part of Fig. 4 .

\section{DISCUSSION}

The significant main effects due to sex are compatible with previously reported research. The effect due to stimulus presence is, at first glance, seemingly supportive of the notion that exhaustive search patterns are employed by Ss. However, the colors used in the present study should have been equally available in memory for matching. Therefore, the main effect

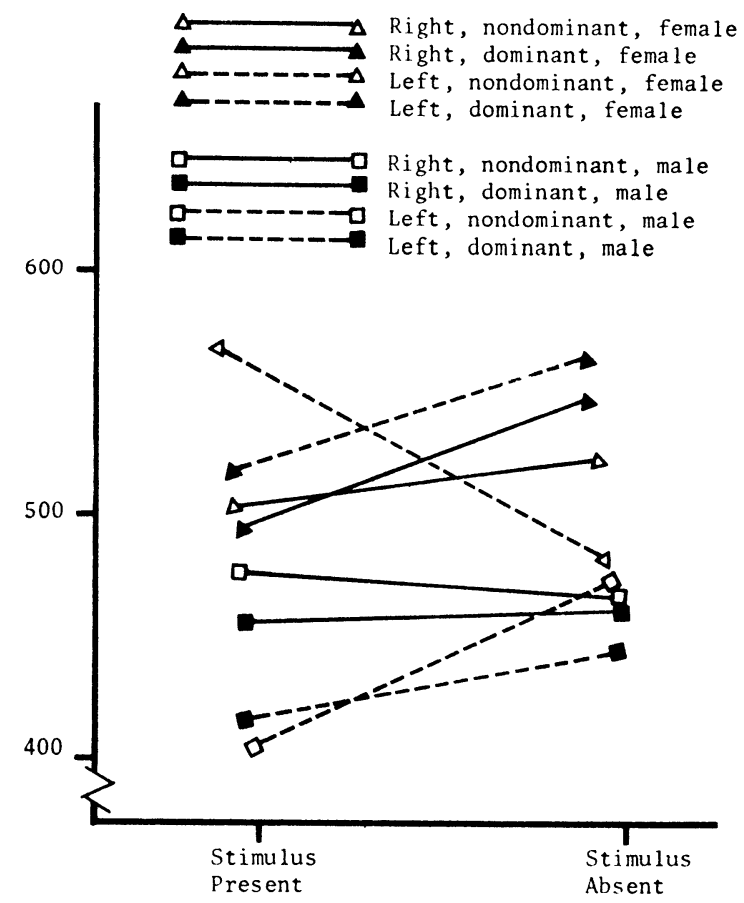

Figure 4. - Mean RT to the presence or absence of probe stimulus in memory set by sex, dominance and handedness. 
due to stimulus presence must be attributed to response selection (faster for positive detection) and not due to search processes. Thus, there seems to be the tendency from most Ss to respond faster to correct or positive stimulus concepts regardless of the availability or location in memory. Perhaps there is a response set generated for the production of responses to positive occurrences of stimulation first. That is, Ss have a higher expectancy of the occurrence of positive stimulation, even though there is no objective evidence for this expectancy.

The results of the present study (see Fig. 1) replicate the interaction between dominance and stimulus presence reported by Dodson (1972). Further, they replicate this interaction utilizing a different RT response (key deflection as opposed to key release) and a different experimental task (color detection as opposed to immediate memory search). Thus, there is reasonable evidence to support the notion that this interaction is a reliable phenomenon.

However, the tentative hypothesis that the interaction is at tributable to responses from right-handed Ss does not appear to be supported. From Fig. 2, 3, and 4, there is indication that the nature of the interaction is more complex than the authors first imagined. Apparently, the interaction is totally attributable to the RTs of left-handed females using their right hands.

A tentative explanation is advanced to account for these interactions. It is proposed that left-handed Ss employ slightly different response selection strategies than right-handed Ss. Furthermore, this response selection bias is amplified in left-handed females. This bias is most probably a function of the difficulty with which left-handed individuals learn to adapt to a predominantly right-handed environment. At the present time, the authors do not offer an explanation to account for this departure from the response tendencies of other Ss. This study is not sufficient to determine the source of this interaction.

Clearly, memory search data from previous studies should be examined for indications of a similar response bias. Since most reports do not indicate and analyze the effects attributable to sex or handedness, these factors should be included in all subsequent research. In addition, the present study should be replicated utilizing a standard memory search paradigm. The interactions found in the present study pose interesting questions with respect to theories and applications of RT as a pure measure of information processing and memory search.

\section{REFERENCES}

Dodson, T. The effect of repitition of memory set items on memory search. Unpublished masters thesis, Vanderbilt memory search.

Rigal, R. Hand efficiency and right-left discrimination. Perceptual \& Motor Skills, 1974, 38, 219-224.

Sternberg, $S$. High speed scanning in human memory. Science, $1966,153,652-654$.

Sternberg, S. Two operations in character-recognition: some evidence from reaction-time measurements. Perception \& Psychophysics, 1967, 2, 45-53.

Sternberg, S. Memory scanning: mental processes revealed by reaction-time experiments. American Scientist, 1969, 57, 4, 421-457.

Theios, J., Smith, P. G., Haviland, S. E., \& Traupmann, J. Memory search is a serial, self-terminating process. Unpublished report, the University of Wisconsin, 1971.

(Received for publication May 31, 1974.) 